\title{
Erratum to: Australian east coast rainfall decline related to large scale climate drivers
}

\author{
Milton Samuel Speer • Lance M. Leslie • \\ Alexandre O. Fierro
}

Published online: 18 May 2010

(C) Springer-Verlag 2011

\section{Erratum to: Clim Dyn}

DOI: 10.1007/s00382-009-0726-1

This is a publisher's erratum for the original publication.

Figures $1 \mathrm{c}, 3 \mathrm{a}-\mathrm{d}$ and $4 \mathrm{a}-\mathrm{h}$ in the original publication of this paper are of low resolution, and Fig. 2 has a scaling error.

The better resolution of Figs. 1c, 3a-d and $4 \mathrm{a}-\mathrm{h}$, and the correct version of Fig. 2 are given below.

The online version of the original article can be found under doi:10.1007/s00382-009-0726-1.

\section{S. Speer $(\square)$}

Faculty of Science, Climate Change Research Centre,

The University of New South Wales, Level 4,

Matthews Building, Sydney 2052, Australia

e-mail: milton.speer@unsw.edu.au

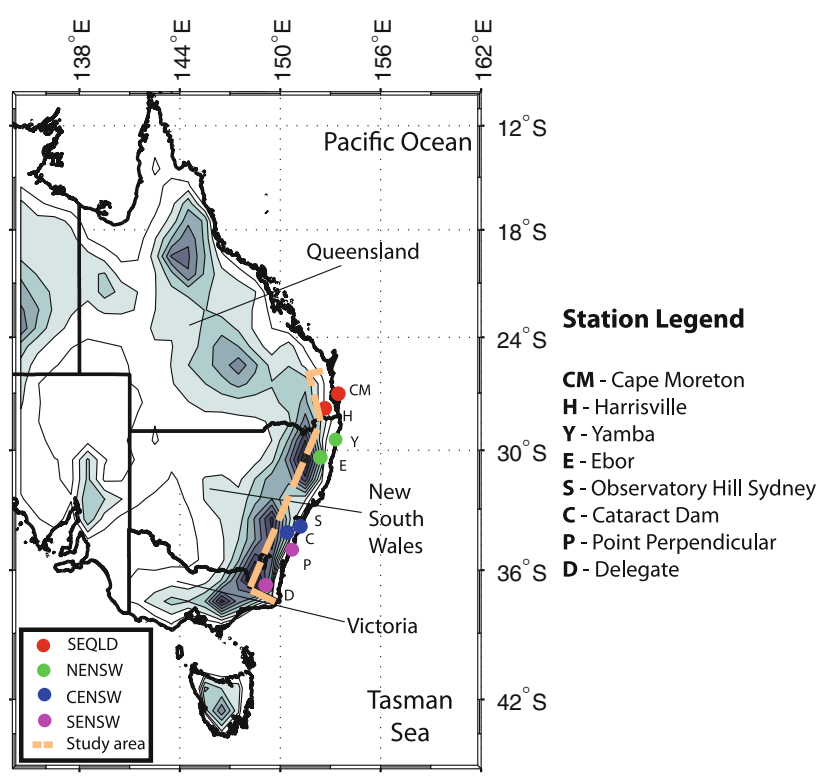

\section{M. Leslie}

Cooperative Institute for Mesoscale Meteorological Studies and School of Meteorology, The University of Oklahoma,

Norman, OK, USA

\section{M. Leslie}

Australian Sustainable Development Institute,

Curtin University, Perth, Australia

\section{A. O. Fierro}

NOAA/Hurricane Research Division,

Atlantic Oceanographic Meteorological Laboratory,

Miami, FL, USA

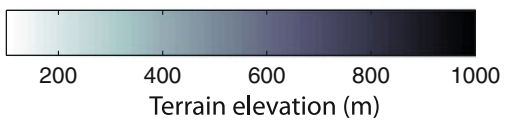

Fig. 1c 
(a) IPO index 1910-2007

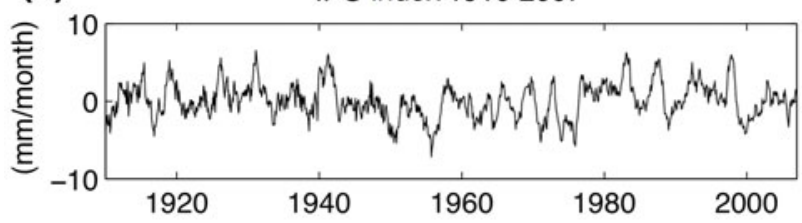

\section{Wavelet Power Spectrum}

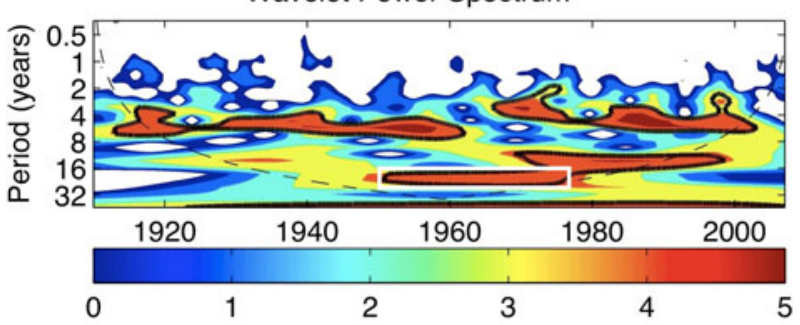

(b) SAM index 1948-2007
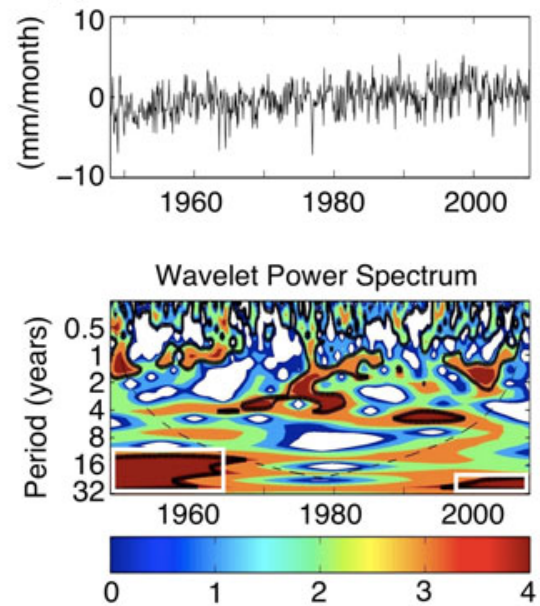

(c)

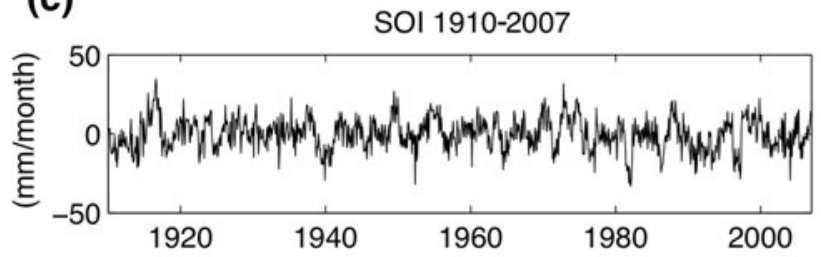

Wavelet Power Spectrum

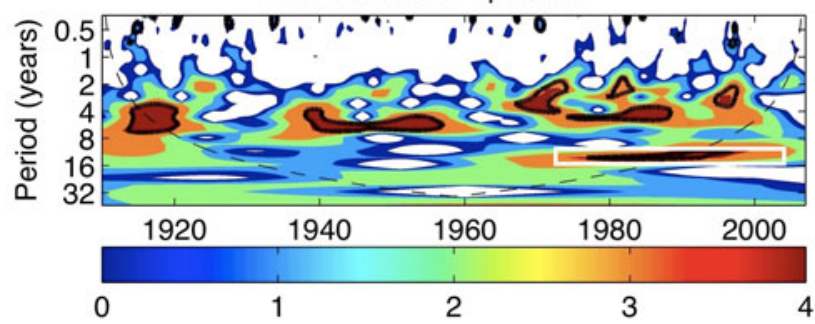

Fig. 2
Global Wavelet Spectrum

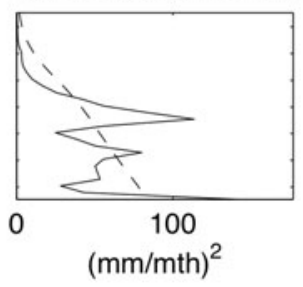

Global Wavelet Spectrum

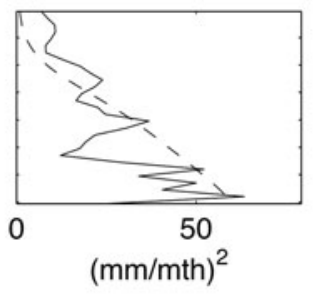

Global Wavelet Spectrum

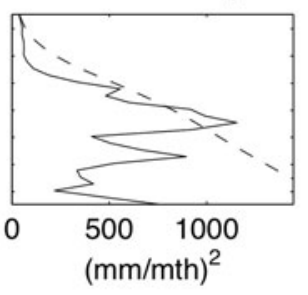


(a)
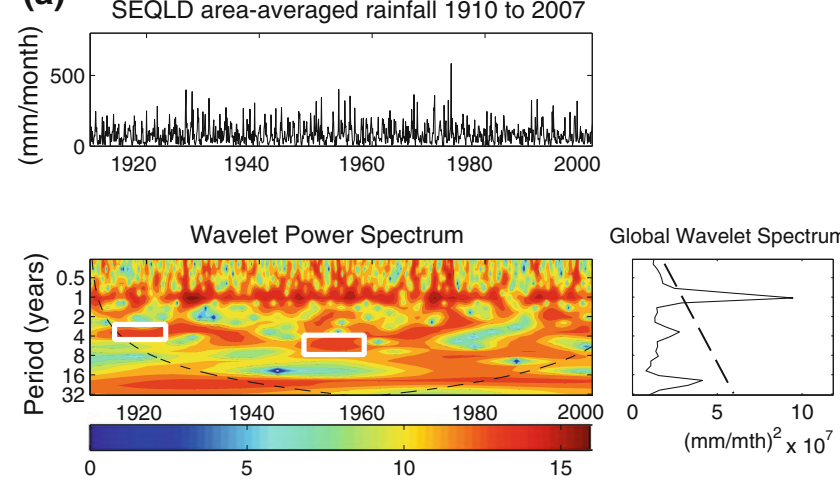

(c)
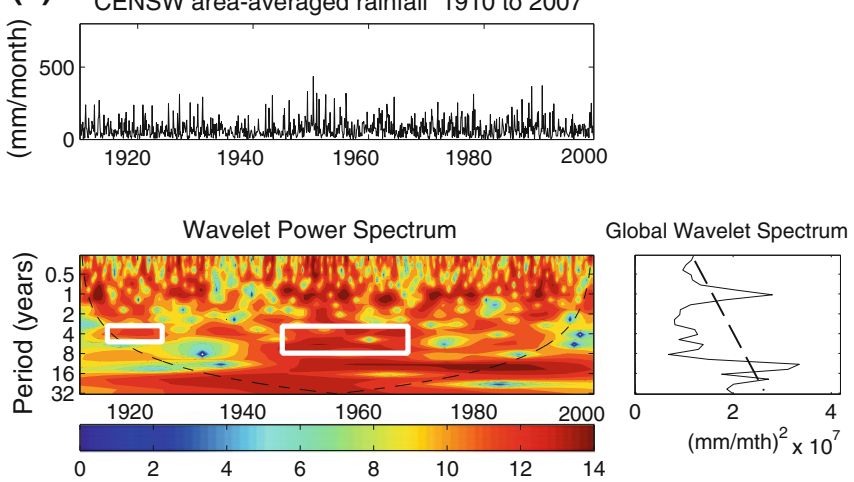

(b)
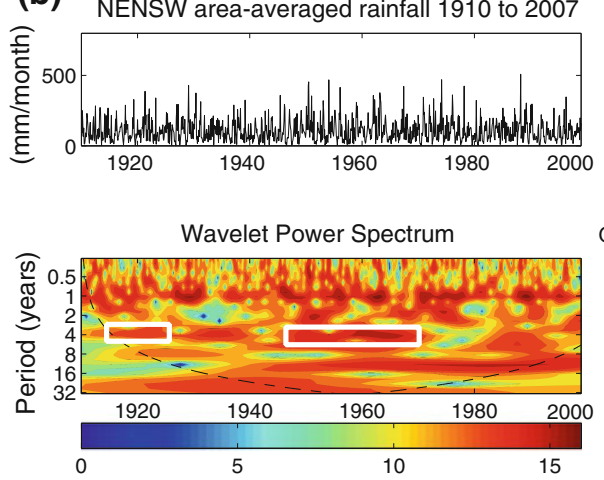

Global Wavelet Spectrum

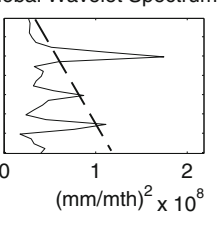

(d)
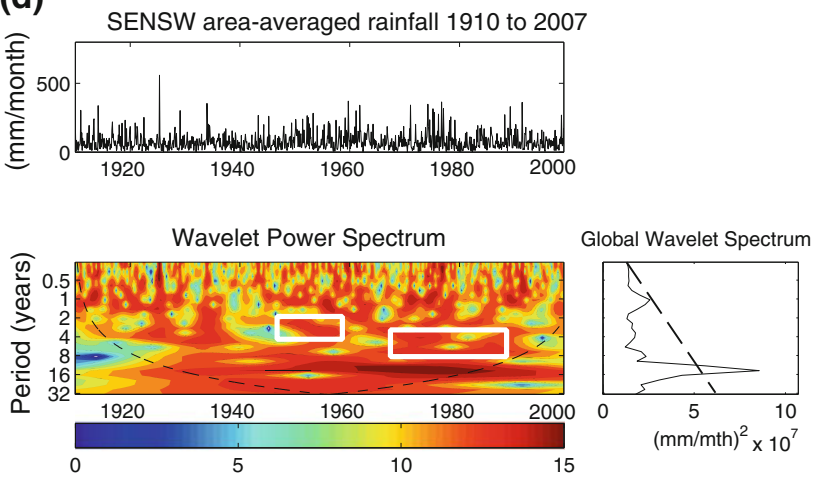

Fig. 3a-d 
(a) Harrisville rainfall 1910 to 2007
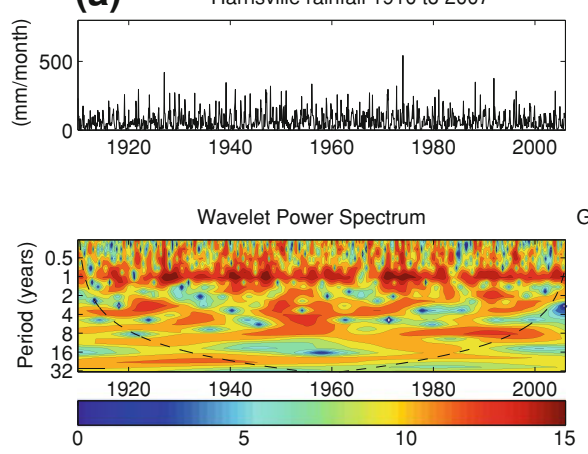

Global Wavelet Spectrum

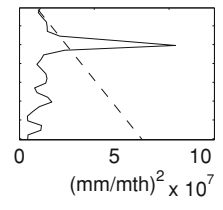

(C) Ebor rainfall 1928 to 2007
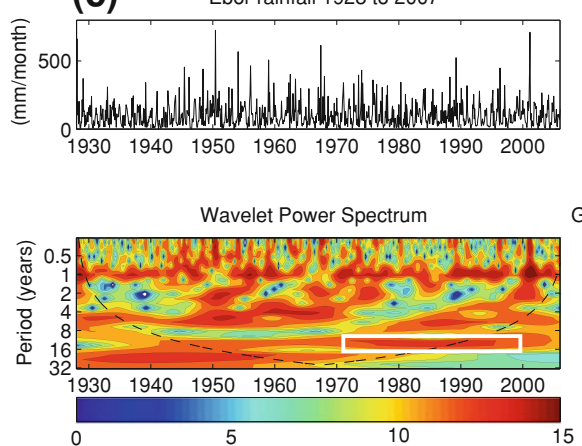

Global Wavelet Spectrum

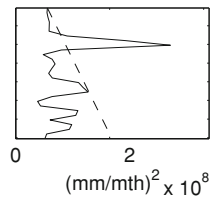

(e)
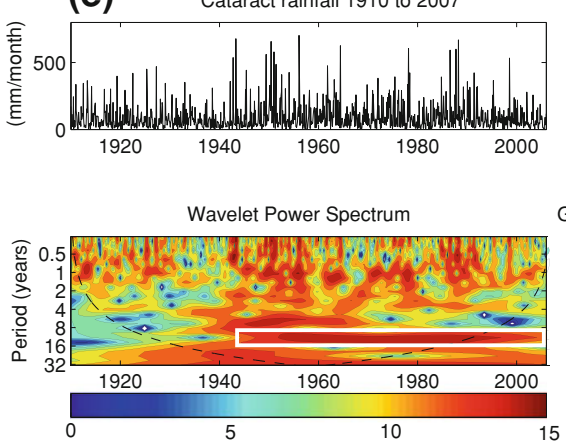

Global Wavelet Spectrum

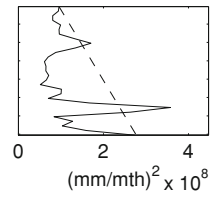

(g)

Delegate rainfall 1910 to 2007
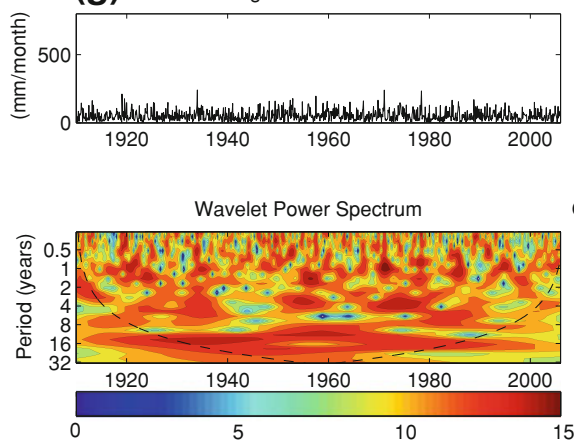

Global Wavelet Spectrum

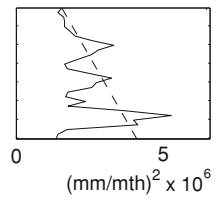

(b) Cape Moreton rainfall 1910 to 2007
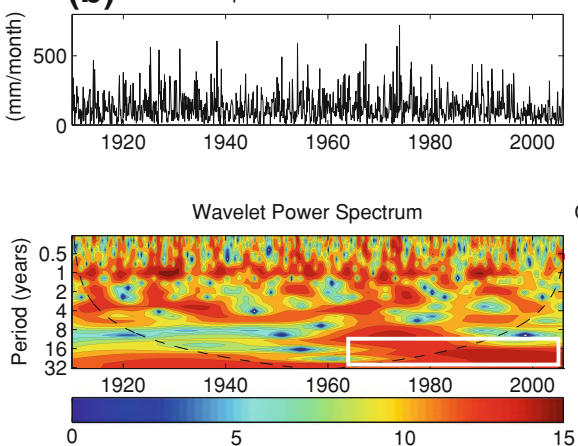

Global Wavelet Spectrum

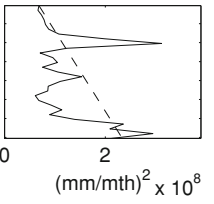

(d)
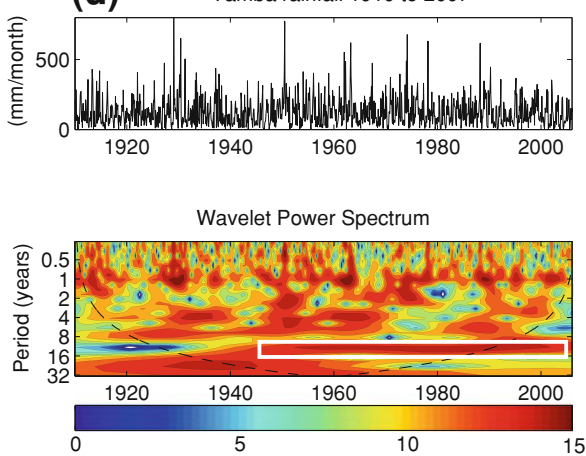

Global Wavelet Spectrum
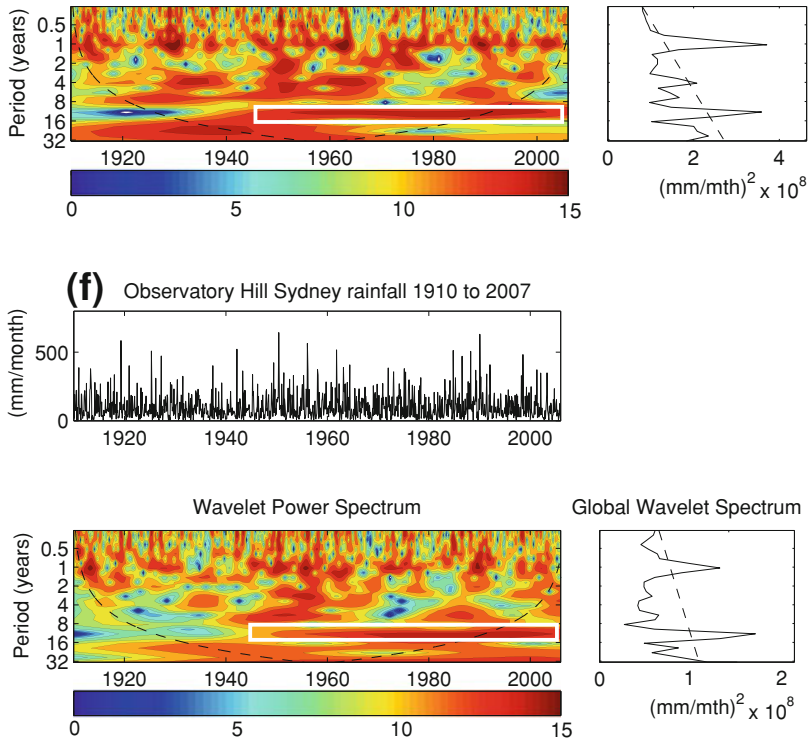

Global Wavelet Spectrum

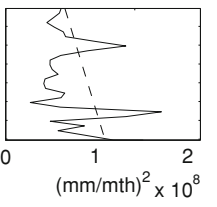

(h) Point Perpendicular rainfall 1910 to 2005
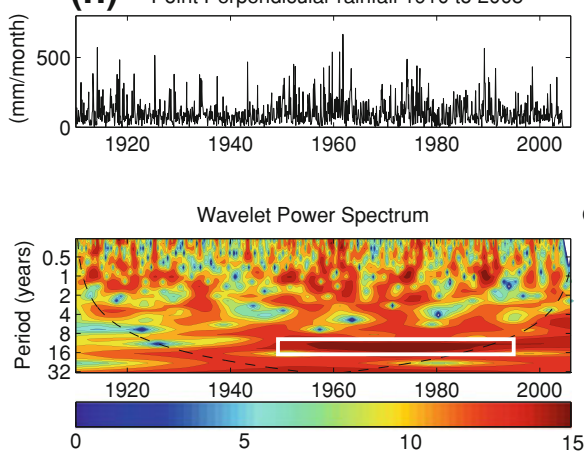

Fig. 4a-h 